\title{
Valoración de la estructura, funcionamiento y financiamiento de organización homóloga a la APROFUNED (Costa Rica) en The Open University (Inglaterra), así como la labor tutorial dentro de un sistema de educación a distancia
}

\author{
Cindy Arias Bogantes* \\ (iD) https://orcid.org/0000-0002-4771-2326
}

Recibido: 09 de abril, 2018 - Aceptado: 06 de junio, 2018

\section{RESUMEN}

Dentro de los objetivos de la Asociación de Profesores de la UNED (APROFUNED), se encuentra el mejoramiento profesional y el fortalecimiento de manera eficiente y efectiva dirigido hacia el trabajo con tutores-profesores de la Universidad. Como parte del cumplimiento de estos alcances, se fomenta el intercambio de conocimientos interuniversitarios, tanto en la parte académica como gremial, por lo que los resultados de la pasantía realizada en The Open University de Inglaterra, giran alrededor de la experiencia y el conocimiento adquirido de asociaciones homólogas, así como de comparar el trabajo que se realiza con el sector docente y valorar opciones de mejora para el beneficio de los tutores en la UNED.

Palabras clave: APROFUNED, tutores, profesores, sindicato.

\section{Formato de citación según APA}

Arias-Bogantes, C. (2018). Valoración de la estructura, funcionamiento y financiamiento de organización homóloga a la APROFUNED (Costa Rica) en The Open University (Inglaterra), así como la labor tutorial dentro de un sistema de educación a distancia. Revista Espiga 17(35), 93-105. Doi: http://dx.doi.org/10.22458/re.v17i35.2092

\section{Formato de citación según Chicago-Deusto}

Arias-Bogantes, Cindy. «Valoración de la estructura, funcionamiento y financiamiento de organización homóloga a la APROFUNED (Costa Rica) en The Open University (Inglaterra), así como la labor tutorial dentro de un sistema de educación a distancia». Revista Espiga 17, n. 35 (junio, 2018): 93-105. Doi: http://dx.doi.org/10.22458/ re.v17i35.2092

* Regente de vida silvestre, especialista en lepidópteros (mariposas) y educadora ambiental. Profesora de la carrera de Manejo de Recursos Naturales, Universidad Estatal a Distancia (UNED), Costa Rica. Máster en Gestión Integrada de Áreas Costeras Tropicales, Universidad de Costa Rica (UCR). Bachiller y licenciada en Manejo de Recursos Naturales, UNED. Bachiller en Economía Agrícola y Agronegocios, UCR, Costa Rica. Correo: carias@uned.ac.cr 


\section{Introducción}

La misión y la visión de la Asociación de Profesores de la Universidad Estatal a Distancia (APROFUNED) está centrada en fomentar el bienestar profesional de los profesores de la Universidad Estatal a Distancia (UNED); también, en promover eventos culturales dentro y fuera de la institución, así como contribuir con el mejoramiento profesional de los profesores a través de seminarios, charlas y actividades sociales desarrolladas por la asociación para sus afiliados.

Por esto, es necesario que sus agremiados y, sobre todo, los miembros de la junta directiva fortalezcan constantemente las competencias y conocimientos para actuar efectiva y eficientemente en pro de su misión y visión. Es por ello que en esta ocasión, se ha creído altamente necesario compartir con asociaciones homólogas a la APROFUNED de universidades a distancia de diferentes latitudes. El propósito de este trabajo gira entonces alrededor de la posibilidad de compartir experiencias con autoridades universitarias, entes académicos, legales y de planificación, así como con tutores y otros académicos, con el fin de analizar estas experiencias y valorar las opciones de adaptar aquellas que puedan ser de alto beneficio para los tutores de la UNED.

Para alcanzar dicho propósito, se escogió The Open University en Inglaterra, ya que tiene similitud con la UNED, sobre todo en cuanto a su modelo de educación a distancia; de igual manera, cuenta con fortalezas en sus sistemas de planificación y un modelo tutorial, con algunas aproximaciones al de Costa Rica, pero con otras fortalezas a nivel gremial y pedagógico. Además, ambas tienen asociaciones o agremiaciones de profesores y, finalmente, porque en la actualidad se cuenta con «convenios marco», elemento que en un futuro cercano podría facilitar el impulso de proyectos de desarrollo y de investigación conjunta.

En cuanto a las funciones de la APROFUNED, se ha centrado la atención de esta pasantía en aspectos puntuales como: el financiamiento de este tipo de asociaciones, las estrategias de proyección interna y externa, el acercamiento y comunicación que estas organizaciones establecen, las normativas técnicas y legales de estas asociaciones con el fin de valorar y mejorar las funciones que actualmente desarrolla la asociación, así como de innovar con nuevas propuesta y proyectos.

En cuanto a la función tutorial, se dirige la atención a las estrategias metodológicas y a las innovaciones tecnológicas y pedagógicas de los sistemas a distancia, de manera que se pueda compartir con los asociados, aquellas estrategias e innovaciones que valoren con posibilidades de adecuarse al contacto.

El mejoramiento de la educación a distancia se verá reflejado mediante el material aportado a tutores-profesores, relacionado con metodologías e innovaciones educativas, las cuales se medirán por medio de las diferentes técnicas y estrategias utilizadas por los profesores-tutores en cada una de las universidades visitadas, tomando como variable de evaluación los criterios de los profesores-tutores sobre los beneficios obtenidos al ser agremiados de la organización, así como las debilidades y oportunidades de mejora. Con la información obtenida, se pretende valorar cuáles aspectos y criterios pueden servir de apoyo e innovación a los tutores-profesores de la UNED. 


\section{Datos generales de The Open University}

De acuerdo con la información suministrada por la encargada de la oficina de relaciones exteriores, Babette Oliver, y del profesor académico Glen Dickinson, ambos de The Open University, esta institución se ubica en Londres, Inglaterra, específicamente en Walton Wal, Kents Hill, Milton Keynes, MK7 6AA, Reino Unido. Además, la información se puede constatar en el sitio web de la universidad ${ }^{1}$.

Su misión es: Promovemos oportunidades educativas y justicia social proporcionando educación universitaria de alta calidad a todos aquellos que desean realizar sus ambiciones y cumplir con su potencial.

A través de la investigación académica, la innovación pedagógica y la colaboración colaborativa buscamos ser un líder mundial en el diseño, contenido y entrega de aprendizaje abierto apoyado.

Su visión es: Los estudiantes son una prioridad en The Open University a través de una estrategia para el crecimiento como medio por el cual continuaremos transformando vidas a través de la educación, aprovechando nuestro éxito y cumpliendo nuestra misión en un mundo cambiante.

\section{Características de The Open University}

The Open Universtiy fue creada en 1969 con modalidad híbrida; desde 2012 se cambió a modalidad 100\% virtual. Actualmente tiene aproximadamente 133600 estudiantes, lo que la convierte en la institución académica con mayor cantidad de estudiantes en todo Reino Unido. Su sede principal está en Milton Keynes, Inglaterra, pero también tiene sedes en Escocia, Gales e Irlanda del Norte.

Por ser una universidad con modalidad del $100 \%$ a distancia, tiene 126000 estudiantes en todo Reino Unido, 1500 en la República de Irlanda, 5000 en otros países de Europa y 1100 en otros países fuera de Europa. La oferta académica es amplia: Grado, maestrías, doctorados, cursos cortos (cursos de formación académica) e investigación académica. Además posee las siguientes facultades: Artes y Ciencias Sociales, Negocios, Leyes, Ciencias, Tecnología, Ingeniería y Matemática, así como Bienestar, Educación y Lenguas.

Perfil del estudiante

El 30\% de los estudiantes tiene menos de 25 años de edad, mientras el $9 \%$ está por encima de los 50 años. La edad promedio es de 25 años. Asímismo, más de 11000 estudiantes estudian en el nivel de posgrado y alrededor de 22000 estudiantes tienen algún tipo de discapacidad. E1 77 \% de los estudiantes graduados en The Open University están dentro del mercado laboral en puestos relacionados con su carrera.

1. Sitio web de The Open University. Acceso: 14 de mayo de 2018, http://www.open.ac.uk/about/main/ strategy-and-policies 


\section{Financiamiento}

El financiamiento se obtiene directamente de las tarifas que pagan los estudiantes anualmente, las cuales dependen del nivel académico en que se encuentren y su nacionalidad. Los montos van entre las 6000 a 9000 libras esterlinas por año (de \$8 000 a \$12000).

Desde el 2012 la universidad no cuenta con financiamiento del Estado, ya que este únicamente brinda colaboración para investigación y becas para estudiantes privados de libertad. Solamente a los estudiantes de Escocia se les exonera la matrícula.

\section{Cantidad de personal}

En total, hay 9375 colaboradores en la universidad, distribuidos de la siguiente manera: Académicos de planta (1062), académicos relacionados o parte de tiempo (1796), tutores, conocidos como Associate lecturers (4982) y administrativos (1535). Estos datos son a octubre de 2017, pero año tras año pueden variar dependiendo de la matrícula.

\section{Estructura organizativa del personal de The Open University}

Con base en la información proporcionada por Oliver y Dickinson, la siguiente es la estructura organizativa del personal de The Open University:

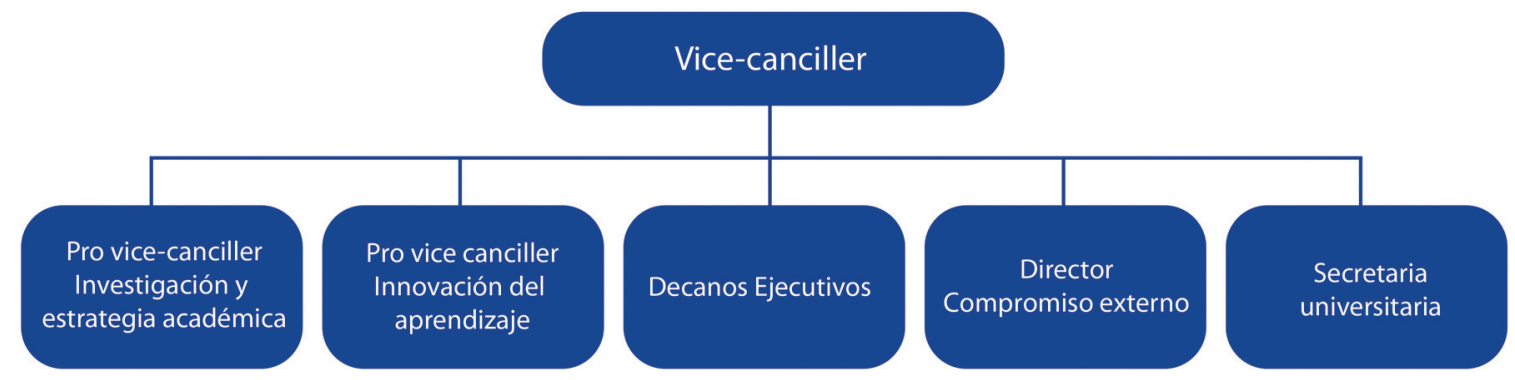

Figura 1. Estructura organizativa del personal de The Open University. Fuente: Oliver y Dickinson, 2017.

El equipo ejecutivo es responsable de las siguientes áreas: Estudiantes; financiera; currículum y reconocimientos; aprendizaje y enseñanza; investigación y emprendimiento; desarrollo de negociaciones; manejo de información universitaria; recaudación de fondos y desarrollo internacional; administración general.

El pro vice canciller de innovación del aprendizaje es responsable de las siguientes áreas: Investigación e innovación digital; liderazgo especializado y apoyo en los procesos de enseñanza-aprendizaje; validación y alcance de actividades; estrategia y planeamiento de recursos académicos.

El pro vice canciller de investigación y estrategia académica es responsable de las siguientes áreas: Estrategias académicas para investigación, currículum y enseñanzaaprendizaje; políticas institucionales y sistemas de calidad; estrategia de liderazgo e implementación del Plan de Investigación Universitario; intercambio de conocimiento y actividades de emprendimiento. 
El director de compromiso externo es responsable de las siguientes áreas: políticas legales; desarrollo de negociaciones; comunicaciones; oficina de desarrollo; mercadeo; asuntos externos y gubernamentales a nivel nacional.

La secretaria universitaria es responsable de las siguientes áreas: Operaciones; servicios académicos; auditorías; políticas académicas y gobernanza; oficinas nacionales (sucursales); oficina de estrategia; servicios de apoyos a estudiantes y tutores; servicios administrativos: finanzas, recursos humanos, información tecnológica.

Los decanos son los encargados de cada facultad (carreras universitarias): Decano de la Facultad de Artes y Ciencias Sociales; Decano de la Facultad de Leyes y Negocios; Decano de la Facultad de Ciencias, Tecnología, Ingeniería y Matemática; Decano de la Facultad de Bienestar, Educación y Lenguas.

\section{Resultados}

Agrupaciones homólogas a la APROFUNED y trabajo con tutores en The Open University

1. Sindicato de trabajadores de The Open University

La siguiente información fue suministrada por Pauline Collins y Caitlin Adams, representantes del Sindicato de trabajadores de The Open University.

El Sindicato representa a todas aquellas personas que laboran para The Open University, ya sean académicos o administrativos. Parte de los objetivos del Sindicato es velar por el bienestar de los agremiados, al brindar soporte en temas como contrataciones, condiciones salariales, aspectos concernientes a la parte profesional o situaciones personales que se presenten.

Los objetivos de trabajo son:

- Ser la principal organización sindical y profesional de todo Reino Unido.

- Negociar a nivel nacional (en la universidad) aspectos salariales y condiciones de trabajo.

- Dar asesoramiento sobre política educativa y planes de pensiones.

- Apoyar a los miembros o asociados en condiciones legales, económicas o sociales.

- Ofrecer cursos de capacitación y desarrollo profesional continuo.

A nivel organizacional, el Sindicato de The Open University se compone de la siguiente manera:

- Comité Ejecutivo: 1 presidente, 2 vicepresidentes, 1 secretaria, 1 tesorero, 1 fiscal.

- 1 oficial de personal académico de la sede central.

- 1 oficial de personal académico de sedes regionales.

- 1 representante de tutores.

Cada año, la Asamblea General Anual elije el Comité Ejecutivo, el cual se reúne cada dos meses; pero si hay casos urgentes por resolver, lo hace cada mes. 
De igual manera, se cuenta con diferentes comités de trabajo, integrados voluntariamente por diferentes miembros asociados. Dentro de los comités están: Comité negociador, grupo de trabajo de tutores, grupo de negociación de tutores, comité de campañas y apoyo profesional (psicólogos, abogados, políticos).

Actualmente, el Sindicato de The Open University cuenta con alrededor de 5000 asociados, entre administrativos, académicos de planta y tutores.

Parte importante del Sindicato es el proceso de negociación, ya que se trabaja con otras universidades públicas a nivel nacional y, cada comisión dentro del Sindicato, es responsable de buscar los recursos, negociaciones y acuerdos necesarios según los objetivos correspondientes. Cada comisión es responsable de trabajar a nivel local y nacional.

A nivel económico, el Sindicato se financia con las cuotas mensuales de los asociados, las cuales se manejan de la siguiente manera: 1 libra esterlina (1,40 dólares o 793 colones, según tipo de cambio del Banco Central de Costa Rica del 23 de febrero, 2017).

\section{TABLA 1}

Cuotas mensuales descontadas a los afiliados del Sindicato en The Open University (montos en libras esterlinas, £, 2017)

\section{Salario anual}

Por encima de 60000

40000-59999

30000-39999

20000-29999

10000-19999

5000-9999

Por debajo de 5000

Fuente: Collins y Adams, 2017.
Cuota mensual (Irlanda del Norte)

Cuota mensual (Gran Bretaña)

28,42

25,80

22,82

20,70

12,89

5,16

1,01

Con respeto a la parte social, el Sindicato no organiza eventos sociales para sus asociados; las actividades sociales que se llevan a cabo en The Open University son organizadas por la misma universidad.

Una de las partes más importantes del Sindicato es el trabajo con los tutores, por lo que se cuenta con dos comisiones encargadas de estos. Entre las principales situaciones o necesidades por trabajar con ellos (Associate lecturers), están:

- Los salarios no corresponden con la carga de trabajo asignado.

- No cuentan con las mismas oportunidades que el personal de planta, como por ejemplo, en actividades, ascensos laborales y acceso a la información.

- Cada año deben hacer el mismo proceso de concurso y contratación, como nuevos oferentes de servicios en la universidad. 
- El tiempo de contratación dentro de cada año es de un 1/8 de tiempo a un 1/4 de tiempo.

Trabajo y funciones de los tutores en The Open University (estructuras representativas de los tutores)

Según información suministrada por Steve Wootton, Toby Scott-Hughes y Denzil DeSouza, del departamento de contratación de tutores, The Open University cuenta con 4200 tutores, los cuales atienden alrededor de 170000 estudiantes de grado y posgrado.

La organización de la contratación y tiempo de asignación de trabajo para los tutores en The Open University, es la siguiente:

- $25 \%$ trabaja tiempo completo (son académicos de planta que también son tutores).

- $39 \%$ trabaja tiempo completo con otra institución o empresa.

- $44 \%$ tiene trabajo de medio tiempo con otra institución o empresa.

- $16 \%$ también trabajan tiempo completo con otra universidad.

- Todos los tutores trabajan con asignaciones de 1/8 a 1/4 de tiempo.

Los tutores y estudiantes trabajan en conjunto mediante la Plataforma Virtual (Student Support Team Moodle). El equipo de trabajo incluye: Personal académico de la facultad, tutores y apoyo de aprendizaje, los cuales en conjunto trabajan en dar apoyo al estudiante en un área del currículum en particular.

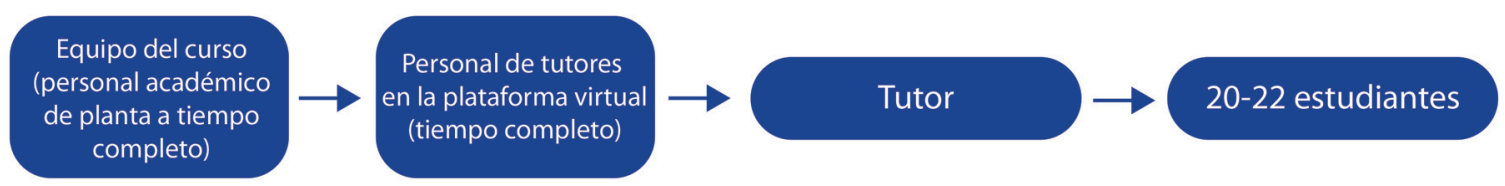

Figura 2. Organigrama estructural de cada curso o asignatura en The Open University.

Fuente: Wootton, Scott-Hughes, DeSouza, 2017.

Dentro de la naturaleza del rol o trabajo de los tutores, se contemplan los siguientes aspectos:

- Encuentro personal con la universidad.

- Apoyo a estudiantes en los módulos (individuales).

- Seguir la línea administrativa del personal docente y académico.

- Seguir el contrato con todos los términos y condiciones del servicio.

- Trabajo bajo la modalidad de teletrabajo.

- Revisar y hacer realimentaciones de las asignaciones, realizar tutorías presenciales (por plataforma) y facilitar actividades en línea. 
El proceso de reclutamiento para trabajar en The Open University como tutor, es el siguiente:

1. Cuando la universidad abre un anuncio o concurso, se debe ingresar a su página web en el link "Enseñando con la OU". El proceso se abre cada setiembre para tener todo listo para el año siguiente.

2. Llenar un formulario en línea.

3. Para algunos módulos o materias se requiere que el tutor-profesor sea sometido a una entrevista previa por parte de los encargados administrativos, con el fin de comprobar el nivel de conocimiento sobre la materia a impartir.

4. Los solicitantes exitosos son invitados a una entrevista, donde se les pregunta sobre aspectos pedagógicos y conocimiento relacionados con el tema.

5. Los solicitantes son informados sobre los resultados 10 días después de la entrevista. Los procesos de contratación y evaluación de los tutores dependen de cada facultad.

Dentro de las funciones de los tutores están:

- Estar listo para iniciar el módulo, el cual es anual y está previamente preparado. Dar la bienvenida a los estudiantes e introducirlos al módulo, explicando aspectos como calificación, asignaciones, forma de tutorías, etc.

- Dar apoyo a los estudiantes de forma reactiva y proactiva, así como trabajar con ellos por medio de la plataforma virtual y atender sus quejas.

- Facilitar el aprendizaje y el desarrollo de las habilidades por medio del contenido académico.

- Monitoreo del proceso de los estudiantes e identificación de sus necesidades.

- Evaluación continua.

- Interactuar con las autoridades universitarias, dar realimentación del proceso del módulo y el trabajo con los estudiantes, así como estar comprometido con el desarrollo de las actividades propuestas por el equipo de trabajo.

Las funciones de los administradores de tutores (personal académico de planta) son:

- Comprometerse con las actividades académicas, investigación y procesos de enseñanza.

- Reclutar, dar inducción, desarrollo y monitoreo de los tutores de la facultad.

- Interactuar en la producción y puesta en marcha de los módulos.

- Investigación pedagógica.

- Evaluar la calidad del trabajo de los tutores.

- Resolver las quejas o necesidades de estudiantes y tutores.

- Dar apoyo a los tutores.

\section{Experiencia como tutor en The Open University}

De acuerdo con la experiencia del profesor tutor Martin Friel, quien ha laborado por 15 años para The Open University, antes de empezar un módulo se debe estar seguro de que todo se encuentre bien, con base en las preparaciones previas de producción realizadas por el equipo académico, así como revisar toda la plataforma con el fin de 
entender con claridad cada una de las partes y la correcta descarga de cada uno de los instrumentos y el material.

Según Friel, las situaciones que se presentan con los tutores son las siguientes:

- Al tener una función totalmente virtual, no se sienten parte de la universidad.

- Se sienten insatisfechos de que no se les dé oportunidad en la elaboración y producción de los módulos y solo se les vea como instrumentos de calificación.

- Deben lidiar con situaciones de estudiantes y no hay apoyo de la parte académica.

- No hay un equilibrio salarial entre el personal académico y los tutores.

- Durante el año y el desarrollo del módulo, se les solicitan trabajos que no están dentro del contrato y no son pagados, por lo que se pierde la perspectiva de tutores.

- No hay oportunidades de ascenso dentro de la universidad.

Según Martil Friel, dentro de los beneficios de trabajar como tutor en The Open University está el ser funcionario de una universidad de mucho prestigio, la flexibilidad de horarios, el sistema de contratos que se tienen (en algunos casos puede beneficiar a los tutores, principalmente aquellos que poseen años de experiencia docente en la institución) y el apoyo que recibe el gremio por parte del Sindicato.

Para tener mejores condiciones laborales y salariales, los tutores deben manejar de tres a cuatro módulos a la vez, pero eso va a depender de la facultad, de la experiencia y de la coordinación académica.

\section{Retos y expectativas para la Asociación de Profesores de la UNED}

Dentro de las oportunidades de mejora, retos y expectativas para la APROFUNED, se mencionan las siguientes:

\section{Trabajo por comisiones}

Se pueden realizar comisiones de trabajo con los mismos tutores asociados, donde cada grupo trabaje con una situación o ámbito en especial. Por ejemplo, se pueden conformar las siguientes comisiones: Eventos sociales, asuntos legales, resolución de conflictos de cargas académicas, de mercadeo y promoción.

¿Cómo ponerlo en práctica y aplicarlo? En las asambleas ordinarias y extraordinarias se deben establecer comisiones de trabajo que trabajen durante todo el año de manera prioritaria como, por ejemplo, la comisión de comunicación y divulgación (que ya existe) o la comisión de asuntos legales (para atender situaciones legales laborales de los asociados), entre otras de acuerdo con las necesidades que se presenten. Las comisiones se pueden reunir una vez al mes para evaluar estrategias de trabajo y analizar resultados y avances; así, las problemáticas de los tutores pueden ser resueltas con mayor rapidez. 


\section{Capacitaciones a tutores en diferentes temas}

La APROFUNED, por medio de profesionales en diferentes áreas, puede brindar asesoramiento y capacitación a tutores en diferentes áreas, tales como charlas, congresos, talleres, etc., con ellas se fomentaría el crecimiento profesional y personal. Entre los temas por abordar están: Planes de pensiones, políticas educativas, administración financiera, carrera profesional.

¿Cómo ponerlo en práctica y aplicarlo? La APROFUNED puede ofrecer capacitaciones periódicas a los tutores (trimestralmente, por ejemplo), las cuales deben fortalecer su calidad y desempeño académico. Estas pueden ser coordinadas con diferentes entidades universitarias, o bien, traer invitados de otras entidades académicas. Los temas pueden ser propuestos por los mismos tutores de acuerdo con las necesidades o deficiencias que se presenten en el sector de profesores. Algunos temas que se pueden trabajar son: diseño curricular, manejo de plataformas en línea, legislación laboral, cargas académicas.

3. Negociaciones con otras asociaciones u organizaciones gremiales de otras universidades estatales

La APROFUNED podría tener convenios o negociaciones con otras asociaciones de profesores u organizaciones gremiales de las demás universidades públicas; esto con el fin de hacer alianzas estratégicas en pro del gremio de los profesores universitarios.

¿Cómo ponerlo en práctica y aplicarlo? La Junta Directiva de la APROFUNED puede coordinar alianzas o estrategias de trabajo con otros grupos gremiales de universidades públicas, con el fin de presionar en ciertos temas, así como fortalecer y apoyar al sector de profesores, indiferentemente de la modalidad de la universidad, necesidades y retos, que pueden ser los mismos. Los grupos, si se fortalecen, pueden resolver situaciones de las universidades públicas a nivel general.

\section{Representantes por zonas regionales}

La APROFUNED debe tener representantes en zonas regionales, quienes se encarguen de evacuar dudas o la resolución de problemas o situaciones que enfrentan los tutores. El representante debe ser asociado y puede haber uno por cada zona geográfica del país para de ese modo abarcar varios centro universitarios como, por ejemplo, Guanacaste, Zona Norte, Caribe, Zona Sur y Valle Central; así los tutores pueden tener un mayor acercamiento a la asociación.

¿Cómo ponerlo en práctica y aplicarlo? Es importante que la APROFUNED logre trabajar de manera activa con los tutores regionales, por lo que se pueden realizar giras de trabajo (se tiene como propuesta ante la Junta Directiva) y establecer grupos o representantes de la APROFUNED en diferentes regiones del país. Estos representantes pueden ser conexión con los demás tutores y un tipo de sucursal para dudas, consultas o resolución de conflictos. Se puede establecer una subcomisión de trabajo por cada región del país. 


\section{Apoyo profesional}

La APROFUNED debe contar con apoyo profesional por parte de sus mismos agremiados. Al aprovechar la formación profesional del asociado, se pueden integrar grupos de trabajo por profesión, por ejemplo, equipos de abogados, de economistas, de psicólogos o de educadores; los cuales no solo pueden ser de gran beneficio para capacitaciones, sino para la atención de los tutores, según sus necesidades.

¿Cómo ponerlo en práctica y aplicarlo? Con el fin de formar grupos para la resolución de conflictos de los tutores, la APROFUNED puede aprovechar las diferentes especialidades y profesiones de los agremiados (abogados, docentes, psicólogos, sociólogos) para que cuando exista una situación crítica, sean los mismos agremiados quienes puedan ayudar a resolverla. Se puede agrupar a los asociados por profesión o especialidad para que ellos mismos formen comisiones de trabajo para la atención de tutores.

\section{Propuesta de cargas académicas por curso}

Como propuesta de modificación en el tema de cargas académicas, se sugiere que a los tutores se les pague por curso y no por cantidad de estudiantes. Además, que se integre cada curso entre 15 a 20 estudiantes y que después de 20 estudiantes se le pague un adicional a cada profesor.

¿Cómo ponerlo en práctica y aplicarlo? Este tema ha sido crítico para la APROFUNED y para las entidades universitarias, por lo que se hace necesario buscar soluciones para el cambio en el sistema de asignación de cargas académicas, de la asignación por horas y la distribución adecuada de trabajo. Actualmente, este tema se trabaja por medio de vías legales, pero es necesario que la Junta Directiva de la APROFUNED se reúna con directores de escuelas y la Vicerrectoría Académica para plantear una posible solución actualizada que se ajuste a la realidad actual del país, de la universidad y de la modalidad virtual, con el objetivo de buscar la estabilidad laboral de los tutores.

\section{Conclusiones}

- El propósito de la pasantía se enfocó en compartir experiencias con autoridades universitarias, entes académicos, legales y de planificación, con de fin de analizar experiencias y valorar opciones que se puedan adaptar y beneficiar a la UNED.

- The Open University tiene similitud con la UNED por su modelo de educación a distancia, por lo que podría facilitar, en un futuro cercano, proyectos de desarrollo y de investigación de manera conjunta.

- Con respecto a los asuntos gremiales, se centró la atención en organizaciones homólogas en asuntos puntuales como financiamiento, proyección, comunicación, normativas legales y trabajo con los agremiados (principalmente tutores), con el fin de buscar propuestas de innovación y mejora de la APROFUNED.

- Se estudió programas de mejoramiento en cuanto a la metodología, innovación educativa, calidad académica, desarrollo y mejoramiento profesional, así como 
herramientas tecnológicas y pedagógicas que se pueden utilizar bajo un modelo de educación superior a distancia.

- The Open University es la universidad más grande de todo Reino Unido, con aproximadamente 133600 estudiantes, con sedes en Inglaterra, Escocia, Gales e Irlanda del Norte; con niveles académicos de grado y postgrado, así como cursos de formación académica.

- The Open University tiene alrededor de 9375 colaboradores entre académicos y administrativos, siendo los tutores profesores el grupo más representativo (aproximadamente 4900).

- Con respecto a las agrupaciones, The Open University trabaja con un Sindicato de trabajadores, el cual representa a todos los funcionarios (académicos o administrativos) y trabaja en temas como contrataciones, condiciones de salario, situaciones personales, planes de pensiones y condiciones legales.

- La asociación o sindicato opera por medio de comités de trabajo (negociación, trabajo con tutores, campañas, apoyo profesional). Cuenta con alrededor de 5000 asociados y es financiada por las cuotas mensuales de sus asociados, según el salario percibido.

- Al igual que en la UNED, en The Open University se presentan los inconvenientes, situaciones o necesidades con respecto a los tutores, como por ejemplo los salarios que no corresponden a las cargas de trabajo asignadas, no cuentan con las mismas oportunidades que el personal de planta o administrativos, cada año o cuatrimestre se debe renovar la contratación, el tiempo asignado de contrato es de $1 / 8$ o un 1/4 como máximo.

- The Open University cuenta con alrededor de 4900 tutores, los cuales trabajan con los estudiantes mediante una plataforma virtual. Se trabaja con un equipo que incluye personal académico de la facultad, tutores y apoyo de aprendizaje. Se trabaja en función de dar apoyo al estudiante en un área del currículum.

- Dentro de las funciones de los tutores están: Introducir a los estudiantes al módulo, calificación de instrumentos, asignaciones, retroalimentación en la plataforma, apoyo a los estudiantes, facilitar el aprendizaje y el desarrollo de habilidades por medio del contenido académico y evaluación continua.

- Como retos que puede tener la APROFUNED en comparación con The Open University, están: Trabajo con comisiones, capacitación a los tutores en diferentes temas, negociación con asociaciones de otras universidades, tener representantes por zonas regionales, apoyo profesional y propuesta de mejora de cargas académicas.

- El objetivo general de The Open University es "El estudiante primero", se pretende brindar una experiencia estudiantil sobresaliente, enfocándose en los objetivos, reclutamiento, retención y calificación.

- The Open University se enfoca principalmente en trabajar con estudiantes mediante intervenciones de preparación por cada módulo, verificación, evaluación, preparación para el estudio, seguimiento, apoyo a los estudiantes y manejo de referencias no relacionadas con apoyos no académicos. 
- Se cuenta con un protocolo de planeamiento para las calificaciones de las asignaciones, por medio de un trabajo proactivo y reactivo con los estudiantes, seguimientos de intervención y procesos de transición dentro de un currículum.

- El diseño curricular es una de las prioridades de The Open University, donde se considera el perfil del estudiante, riesgos y cultura, tomando en consideración un diseño que sea conveniente y flexible, en el cual se lleve a cabo un proceso de enseñanzaaprendizaje y desarrollo de habilidades.

\title{
SUMMARY
}

Assessment of the infrastructure, functioning, and financing of The Open University, England's counterpart to APROFUNED (Costa Rica), as well as the tutoring work in a distance education system

\begin{abstract}
Some of the objectives of UNED's Association of Teachers (APROFUNED) are the improvement of its workforce and the efficient and effective strengthening of their tutoring and teaching practices. As part of the fulfillment of these efforts, an interuniversity exchange of knowledge is fostered both in the academic and union sectors. The results of the internship at England's The Open University revolve around the experience and knowledge acquired from counterpart associations, as well as the possibility to compare the work done with the education sector and to assess options for the improvement of UNED's tutors.
\end{abstract}

Key words: APROFUNED, tutors, professors, unions.

RÉSUMÉ

Évaluation de la structure, du fonctionement et du financement de The Open University, à l'Anglaterre, organisation homologue à l'APROFUNED au Costa Rica, ainsi que du travail des tuteurs dans un système d'enseigenement à distance

Parmi les objectifs de l'Association des Professeurs de l'Université de l'Etat à Distance (APROFUNED) se trouve l'amélioration profesionnel et le renforcement efficace et effective du travail des tuteurs de l'UNED.

Dans le cadre de l'accomplissement des buts, l'echange des connaissances interuniversitaires (académiques et de l'organisation profesionnelle) est favorisée ; c'est pourquoi les résultats du stage realisé en Open University à l'Anglaterre tournent autour de l'expérience et les connaissances adquises sur les associations homologues, de la comparaison du travail qui fait le corps enseignant et de la mise en valeur d'options d'amélioration au bénéfice des tuteurs de l'UNED.

Mots-clés: APROFUNED, tuteurs, enseignants, syndicat.

\section{Bibliografía}

Sitio web de The Open University. Acceso el 14 de mayo de 2018, http://www.open.ac.uk/about/main/ strategy-and-policies 